\title{
Interactive comment on "Analysis of deformation bands associated with the Trachyte Mesa intrusion, Henry Mountains, Utah: implications for reservoir connectivity and fluid flow around sill intrusions” by Penelope I. R. Wilson et al.
}

\section{Laurel Goodwin (Referee)}

laurel@geology.wisc.edu

Received and published: 18 August 2020

\section{General Comments}

This beautifully illustrated paper is divided into two parts: (1) a description of deformation band networks associated with the Trachyte Mesa intrusion and analysis of their physical significance and (2) an evaluation of the impact of these networks on fluid flow. This overview addresses each part, then considers the paper as a whole. Specific comments re: figures and text follow. 
The core of this manuscript is a description of the orientations and detailed analysis of the topology of deformation band networks that are demonstrably physically associated with the Trachyte Mesa intrusion. The methods used, results presented, and limitations of data provided in this section of the paper are remarkably clear, honest, and well documented. I was particularly pleased to see a specific, clear, and quantifiable description of terms such as 'intensity' that are commonly used loosely to mean several different things. The authors note that quantitative assessment of network topology was undertaken only on surfaces oriented at a high angle to the average strike of deformation bands documented by previous studies - a reasonable choice of approach to maximizing information acquired given likely constraints on time and outcrop. The results of this work are compelling. In my opinion, they represent the most significant contribution of the manuscript, showing that deformation bands increase in abundance, length, and connectivity across the margin of the intrusion. I agree with the authors that quantitative studies such as this "are essential to improve and constrain laboratory and numerical models of intrusion emplacement mechanisms and associated deformation." The only substantive criticism I have of this portion of the manuscript is the use of the term 'fracture' to refer to deformation bands. I know that small-displacement fractures and deformation bands are sometimes lumped together but disagree that such lumping is constructive. A fracture is a surface across which cohesion has been lost; the same is not true of a deformation band, which is a zone of deformation within which fractures may or may not form. I therefore suggest modifying the text to state that topological analysis of fractures can be extended to other discrete structures and in this case has been applied to deformation bands. Subsequent references to the structures studied should consistently refer to them as deformation bands.

In addition to the detailed analysis of attributes of the deformation band network, the authors include a qualitative assessment of porosity and permeability of the deformation bands. In contrast to the evaluation of geometry of deformation band networks, this analysis is neither rigorous nor well documented. Porosity was estimated for host rock in one thin section from each field station and for deformation bands in thin sections 
from the four stations that included them. Estimates were made by visual comparison with charts in a sedimentary petrology textbook. Data are given in a plot (Fig. 7), with a single value for deformation band porosity and a range of values for host rock porosity. We are not told what magnification was used for analyzed images, how many images were analyzed (does the fact that a range of porosity is not reported for deformation bands indicate that only one image was used to evaluate deformation band porosity in each sample, or that porosity is surprisingly constant in deformation bands?), or what size area was analyzed for each sample. We are not told if the authors took the time to determine an appropriate representative elemental area for these samples. [Porosity will vary within a sample; however, the average porosity of multiple measurements will level off when a representative elemental area is attained.] The use of visual comparison charts is also an issue. In my experience with students using such charts in petrography labs, this qualitative analysis of the same area by different individuals can vary as much as $10 \%$. This is not an accurate approach to analyzing porosity. In my opinion, a more rigorous analysis is necessary to attain publishable data. Point counts are always an option. I suspect, however, that it would be faster and more accurate to download ImageJ, analytical freeware that is commonly used for this purpose (in addition to analysis of such variables as percent cement or grain size distribution), from this site: https://imagej.nih.gov/ij/download.html. Image processing to (for example) separate out blue areas that represent pores can facilitate analysis.

The approach the authors used to infer permeability reduction associated with deformation band formation - "assuming Kozeny-Carmen equation fundamentals" (line 341) is not clear. The equation was developed to estimate permeability in sediments based not only on porosity, but also on grain size and sphericity (both of which change in cataclastic deformation bands). How were they able to extract relative permeability by 'assuming' these 'fundamentals'?

In sum, the authors present a beautifully illustrated and detailed quantitative analysis of deformation band networks observed in a transect across the margin of an igneous

Printer-friendly version

Discussion paper
Interactive

comment

\section{列}


body intruded as a series of sills. They then present an intriguing qualitative analysis of the hydrologic significance of these structures. Data collection, analysis, and interpretations in the latter portion of the paper require some revision for accuracy, clarity, and rigor, after which I recommend publication.

\section{Specific Comments Linked to Figures}

The authors indicate that the outcrops studied are all part of "massive" sandstone roughly $10 \mathrm{~m}$ thick, implying that the samples collected are all part of a single host rock unit. The term "massive", however, is applied by sedimentologists to strata that are structureless, either from the time of deposition or due to post-depositional processes such as bioturbation. However, it is evident from the images and descriptions of sedimentary features provided by the authors that the outcrops studied are neither structureless nor uniform. Figure 2 beautifully illustrates both lateral and vertical variations in sedimentary structures, as well as subtle differences in color and resistance to weathering, consistent with variations in grain size and/or cement mineralogy or percent. In addition to removing the term 'massive' from the paper, I propose the authors explicitly state that although it is not possible to trace a single bed across the margin of the intrusion, their analyses suggest they have sampled rocks with relatively similar grain size, grain rounding, and mineralogy.

Figure 8 is very attractive, but not designed for ease of understanding. I'm a microstructure geek, and I found it hard to navigate because part of the information that would normally be provided in the caption of a single image is given in the text, some is in the caption, and some is beneath a single figure. Some of the labels on images are very difficult to see. For example, I searched for Fe labels after I saw in the caption that Fe referred to 'iron staining' (staining of what? does this mean iron oxide grains or cement or coating?). The dark text does not show up on dark background. Red labels are hard to see; DB labels should be backed with white boxes to stand out and arrows generally need to be larger (only the TMFS- 6 arrows really stand out). In general, it would be better if labels were bold; those imposed on dark areas of thin sections should be white. In

Printer-friendly version

Discussion paper 
short, it is not possible to glean all of the important information about an image from the figure and caption alone. Because the data acquired from thin sections are important to this story, I suggest a different approach. Move the partial captions beneath each image into the main caption and add information. For example: TMFS-1 ( 20 porosity), TMFS-2 ( $\sim 15-20 \%$ porosity), and TMFS-3 ( $\sim 30-35 \%$ porosity) are all well sorted, subrounded, subarkoses with local poikilotopic calcite cement. Only TMFS-3 includes deformation bands. Porosity is reduced to $<5 \%$ in the deformation band, within which small, angular grain fragments provide evidence of limited cataclasis (example highlighted with a bold arrow). Walk the reader through the rest of the photomicrographs in a similar way. Be sure to clearly state what you see as well as what you infer. You don't see pressure solution; you infer it from embayments in grains at point contacts (which can be better highlighted with bold arrows). You don't see cataclasis, you infer it from angular grain fragments. You don't see compaction; you infer it from reduced porosity and preferred alignment of elongate grains (which you don't mention anywhere, but should). In other photomicrographs you can see alignment of elongate clasts parallel to cross laminae or deformation bands. It's good to point that out. Also, I personally like the fact that you haven't drawn lines over deformation band boundaries. For readers less familiar with what these features look like, you may wish to provide some guidance in either words or arrows that mark top and bottom boundaries to a band.

You note 'indistinct "fuzzy" boundaries to larger grains' beneath your last photomicrograph. Most of the grains are quartz and have sharp margins. Your labeled plagioclase grain has "fuzzy" margins, which are also locally brownish in color. Without being able to zoom in further or look at this on an SEM, I would say that there are several things that could contribute to this appearance. Top on my list is margins that are oblique, rather than perpendicular, to the surface of the thin section. Where the edge of a grain dips away from the grain center, it will be increasingly out of focus with distance. With extensive cataclasis, you may be looking through a zone of fine grain fragments on that grain edge. I think this is what you are referring to, but I'm not sure. If it is, spell it out and highlight the specific margin. If I were you, however, I would focus on more obvi- 
ous evidence of cataclasis: a high percentage of angular grains that are substantially reduced in size with respect to subrounded grains evident in host rock.

On p. 8, you also discuss 'early development of sub-grain boundaries', and follow that on p. 9 with observations of 'clear sub-grain boundaries parallel to deformation band orientations.' In general, we use the term 'subgrain' (with no hyphen) to refer to a part of a larger grain separated from the host grain by a dislocation wall. Production of subgrains is part of the process of rotation recrystallization; it is not a brittle process. Subgrain boundaries are only visible with crossed polars, which causes differences in orientation of the crystal across these dislocation walls to show up as differences in exteniction (grayscale). The only features I see oriented subparallel to deformation bands appear to be cracks. Please revise the text for clarity and accuracy.

\section{Specific Comments re: Text and Interpretations}

Add a reference to the list of studies of deformation band impacts on flow (line 40): Sigda, J.M., Goodwin, L.B., Mozley, P.S., and Wilson, J.L., 1999, Permeability alteration in small-displacement faults in poorly lithified sediments: Rio Grande rift, central New Mexico: In Haneberg, W.C., Mozley, P.S., Moore, J.C., and Goodwin, L.B. (eds) Faults and Subsurface Fluid Flow in the Shallow Crust, AGU Monograph 113, 51-68.

Change lines 51-53: "Deformation bands preferentially form in more poorly lithified layers within quartz arenite to arkosic sandstones (i.e. those lacking in lithics) at shallow depths (1-3 km; Fossen, 2010)" to: "Deformation bands within quartz arenite to arkosic sandstones (i.e. those lacking in lithics) preferentially form in more poorly lithified layers at shallow depths (1-3 km; Fossen, 2010)." The former suggests deformation bands are restricted to poorly lithified layers of specific composition.

In lines 195-196, the authors refer to 'cycles enclosing blocks' and note common features of 'networks with lots of cycles'. The discussion of cycles refers to Figs. $6 \mathrm{c}$ and $2 b \& c$, but it is not possible to understand how the reader is supposed to connect this information to the images. The term 'cycle' is not defined, and it is never mentioned 
again. If it is important to the story, the authors should define what they mean and why it is relevant. If it is not, they should remove references to 'cycles'.

In line 218, the authors refer to 'a slightly coarser grained bed within the sandstone horizon'. I am not aware of a definition of 'horizon' used in this context. It appears to be a way to suggest associations between samples collected. Does it refer to the $4 \mathrm{~m}$ thick section of sandstone shown in Fig. 2? Please clarify.

Interactive

comment

I would like to see the authors replace references to 'weak' deformation or cataclasis with more specific information regarding observations rather than interpretations. I suspect they mean that evidence of fracture and associated grain-size and porosity reduction is present, but not as extensive as in other samples, as suggested by higher estimates of porosity.

I suggest the authors replace 'grain crushing' with 'distributed microcracking' in places like line 233. I think it is a more accurate representation of the variable amounts of grain-size reduction via fracture illustrated in their thin sections. Their photos show a range from deformation bands in which the majority of grains are subrounded and similar in size to those in the host rock to deformation bands in which most of the grains have been reduced to relatively small angular fragments and relatively few original grains remain.

On line 235, replace 'Calcite is also present' with 'Calcite locally fills pores'.

Line 245 refers to early development of subgrain boundaries. I addressed misconceptions re: subgrain boundaries in the previous section on Specific Comments Linked to Figures. The authors should make appropriate changes to the text here also.

On line 248, the authors discuss embayed contacts. I think it would be helpful to clarify what is meant by 'embayed', with reference to more clearly annotated examples in thin section images.

Printer-friendly version

The sentence beginning on line 256 states that 'Haematite is also incorporated into 
the matrix within deformation bands as a result of quartz grain crushing. Note the brownish-staining of deformation bands in Figs. 7a and 8". What evidence supports this interpretation? Is it possible that hematite was precipitated after formation of deformation bands? Please provide evidence (. . . and you don't need to hyphenate brownish \& staining).

Interactive

Line 305 refers to 'minor cataclasis as evidence for shear'. Minor cataclasis can occur by compaction alone. It doesn't require shear.

On line 309, the authors propose that evidence of compaction in sandstone suggests confining pressure may increase with proximity to the intrusion. It is certainly a sign of shortening, consistent with intrusion, but that suggests an increase in marginperpendicular stress, not an increase in confining pressure. Note also that intrusions, particularly shallow crustal intrusions, cool very rapidly. The temperature gradient between thin sheets of partially crystalline magma and wall rock so shallow it still has high porosity is very high, and temperature dissipates rapidly at cool shallow temperatures. If you know the thickness of individual sills and likely depth of intrusion, you can do a back of the envelope calculation to determine the cooling rate for a normal geothermal gradient (or even a slightly elevated one, which would not produce high temperatures at relatively shallow depths where you see high porosity sandstones). 'Pressure solution' actually has nothing to do with pressure. It is caused by a stress-induced chemical potential gradient. I suspect that what you are seeing is that the deformation bands that have accommodated the greatest deformation have the highest number of high-stress point contacts between grains, where solution mass transfer is facilitated.

Line 311: Crush breccias do not consist of fragments that are only visible with a microscope. You do show clear evidence of cataclasis, which could be defined as distributed microcracking and rotation and translation of resulting clasts. You might want to provide a definition like this where you first introduce the term in the paper, to facilitate discussion here.

Printer-friendly version

Discussion paper 
Lines 313-314: If a principal slip surface or fault core were present, you would call it a fault or a deformation band fault and not a deformation band.

Line 317: I am not familiar with the term 'permeability pathway'. Deformation bands are features that can influence flow pathways, but they cannot be considered in isolation. In this case, the elephant in the hydrologic room is the extremely low permeability intrusion. Regional flow will take the 'easiest' route around the intrusion, which will be influenced not just by deformation band distribution and connectivity but also by the permeability of the surrounding undeformed rocks. This paragraph also reflects a misunderstanding of the hydrologic significance of microstructural observations. The fundamental misapprehension is that tabular structures that formed by different processes (e.g., compaction bands vs. cataclastic deformation bands) can influence flow differently even if they have the same permeability.

Your intrusion is effectively an impermeable wall. Your deformation band networks may redirect or inhibit flow in a shell around the plutons, or the main effect may be created by the intrusion itself. The best way to determine these effects would be to measure the permeabilities of cores cut in different directions through the networks, then work with a hydrogeologist to model flow. Without those data, I think you are restricted to providing a clear description of the structures at different scales. Please appreciate that the description itself is a significant contribution.

Lines 327-328: I do not know if anyone has published evidence of magmatic fluids of appropriate composition to precipitate calcite. I think this would be a more compelling suggestion if the authors could cite a study indicating it was possible. I do know that the solubility of calcite decreases with increasing temperature, so I suspect that heat introduced by the intrusion could facilitate precipitation of calcite from surrounding groundwater of appropriate composition.

Printer-friendly version

Lines 335-336: I think it is particularly important to replace 'fractures' with 'deformation bands' in these sentences.

Interactive

comment

Discussion paper 
Line 342: I don't know what the authors mean by "However, this assumes a homogeneous development of the grain-scale processes". Please explain.

In line 351, the authors state "At Trachyte Mesa, deformation bands decrease markedly from $\sim 5$ to $10 \mathrm{~m}$ above the intrusion margin.." I assume they mean deformation band intensity decreases. However, they do not present data showing vertical variations in deformation band networks. Is this a personal observation? If the authors have data that show this variation, they should provide it. If relevant data have been published, they should cite the reference.

I suggest the authors modify lines 354-357 to state: "In addition to reducing the bulk permeability of the reservoir, the deformation bands largely strike parallel to the intrusion margin (Wilson et al., 2016), producing an anisotropy in permeability similar to that of a fault zone (e.g. Farrell et al., 2014).

Line 360-361 should be modified to state: 'Gaining a better understanding of these emplacement-related deformation structures may have important implications for fluid flow, hydrocarbon reservoir connectivity / deliverability, hydrology, geothermal energy and $\mathrm{CO} 2$ sequestration...'

I suggest the authors modify line $378-379$ as follows: "The increase in margin-parallel $\mathrm{Y}$ - and $\mathrm{X}$-nodes with proximity to the intrusion is likely to inhibit flow perpendicular to the intrusion margin, as well as potentially forming non-producible reservoir zones."

Interactive comment on Solid Earth Discuss., https://doi.org/10.5194/se-2020-71, 2020. 